\title{
Heterodyne ECE diagnostic in the mode detection and disruption avoidance at TEXTOR
}

\author{
A. Krämer-Flecken ${ }^{1,3, a, b}$, K.H. Finken ${ }^{1, a}$, V.S. Udintsev ${ }^{2, a}$, \\ H. Larue ${ }^{3}$ and the TEXTOR team \\ ${ }^{1}$ Institut für Plasmaphysik, Forschungszentrum Jülich GmbH, EURATOM Association, \\ 52425 Jülich, Germany \\ ${ }^{2}$ FOM-Instituut voor Plasmafysica 'Rijnhuizen', Association EURATOM-FOM, \\ PO Box 1207, 3430 BE Nieuwegein, The Netherlands \\ ${ }^{3}$ Zentrallabor für Elektronik, Forschungszentrum Jülich GmbH, 52425 Jülich, Germany \\ E-mail: a.kraemer-flecken@fz-juelich.de
}

Received 6 December 2002, accepted for publication 10 July 2003

Published 3 November 2003

Online at stacks.iop.org/NF/43/1437

\begin{abstract}
The paper describes the detection of the $m / n=2 / 1$ disruption precursor at TEXTOR. This frequently observed precursor is detected by realtime cross-correlation of two radially separated channels of the heterodyne electron cyclotron emission diagnostic. An electronic module developed to detect the precursor and its functionality is briefly described. A disruption statistic for TEXTOR is presented, from which the probability of the occurrence of the $m / n=2 / 1$ mode is seen. A method to generate reproducible disruption precursors at TEXTOR is also presented. Experiments to avoid disruptions by inducing toroidal velocity shear by tangential neutral beam injection and mitigate disruptions by He-puffing are presented. In the experiments performed, up to 50\% of the disruptions could be avoided. Furthermore, calculations of the precursor frequency and its evolution are described and compared with measurements.
\end{abstract}

PACS numbers: 07.57.Pt, 52.30.Ex

\section{Introduction}

Disruptions are major concerns in the operation of tokamaks. Especially for the next generation of fusion devices such as ITER, with its large stored energy content, an urgent need for methods to detect disruptions exists. During disruptions large forces act on the tokamak vessel and its interior parts, and the large amount of plasma energy deposited on the first wall components [1] within a few milliseconds causes serious damage. Therefore, disruptions should be avoided or mitigated in general. One way to avoid disruptions is the operation of tokamaks in a regime that is easy to handle from the control point of view. However, the operation in advanced scenarios or improved confinement modes is very complicated and deviations of a control parameter from the pre-programmed optimal value can lead to a disruption. In this case, a method should be available to detect early sign of disruptions and mitigate or, even better, avoid the energy quench by appropriate means. In general, there are two ways to predict a

a Partners in the Trilateral Euregio Cluster.

b Author to whom any correspondence should be addressed. disruption: (i) with the help of a neural network [2-4] a control computer is trained to recognize deviations from several input parameters (ten or more); (ii) finding a common disruption symptom, which can be detected by appropriate means. The first method requires a large amount of data from disruptive and non-disruptive plasmas to train the network. With the amount of the data used for the training of the network the probability and reliability for the disruption forecast increases. The training has to be repeated if the neural network is transferred to another fusion device. The second method does not depend on a special training and can be transferred easily to other devices with only small adjustment of the cross-correlation thresholds. Only two signals are included in the processing and the probability of the detection of the precursor is rather high.

At TEXTOR, we developed a method to detect the disruption precursor based on an electronic module for the online calculation of the cross-correlation coefficient. This module is integrated into the plasma control system [5]. The detection method was tested at TEXTOR together with tangential neutral beam injection. With the tangential injection the toroidal plasma rotation profile and its gradient increases. 
A steep rotation gradient across the island will tend to tear apart the $m=2$ mode and increase the thresholds of the instability limit [6]. Also, gas puff experiments with He were performed together with the detection module to mitigate the disruption effects, especially to suppress the generation of runaway electrons [7].

The paper is organized as follows. In section 2, the set-up at TEXTOR is explained, as well as the method to detect the $m / n=2 / 1$ modes in TEXTOR. Section 3 is devoted to the reliability of the detection of disruption precursors. Section 4 is divided into four subsections. The first one describes the mode generation. Section 4.2 is devoted to disruption suppression in Ohmic target plasmas and section 4.3 discusses experiments with disruption suppression by neutral beam induced velocity shear. Furthermore, a comparison of the calculated and the measured plasma rotations is presented. Section 4.4 presents the disruption mitigation with He gas puff. In section 5, the results obtained are summarized and an outlook on future experiments is given.

\section{A method to detect the $m / n=2 / 1$ disruption precursor at TEXTOR}

TEXTOR is a medium size tokamak $\left(R_{0}=1.75 \mathrm{~m}, a=\right.$ $0.46 \mathrm{~m}$ ) with a circular plasma cross-section and a toroidal belt limiter. TEXTOR is well equipped with an electron cyclotron emission (ECE) diagnostic [8]. A 11-channel ECE diagnostic with a bandwidth of $100-200 \mathrm{MHz}$ and a sample frequency of $10 \mathrm{kHz}$ is used for the measurement of electron temperature profiles. The radial resolution is $\Delta r \leqslant 0.01 \mathrm{~m}$. The system operates in the frequency range $105 \leqslant f \leqslant 145 \mathrm{GHz}$ and covers two-thirds of the plasma diameter for standard operation at $B_{\mathrm{t}}=2.25 \mathrm{~T}$. It is coupled via a $\mathrm{x}$-band wave guide to a long horn antenna and a parabolic antenna. The horn antenna is placed on the low field side (LFS) and the parabolic antenna on the high field side (HFS). Those ECE radiometers having their observation volume on the LFS, for standard plasma parameters, are connected to the horn antenna, and the others to the parabolic antenna. The observation of HFS and LFS with the two antennae is a unique feature at TEXTOR. In addition, several ECE spectrometers $(1 \mathrm{MHz}$ sampling, $400 \mathrm{MHz}$ bandwidth) are operated for the study of fast processes and MHD studies. The spectrometers observe the plasma via large elliptical mirrors. The beam waist is less than $0.01 \mathrm{~m}$ and also allows the observation of temperature fluctuations.

At TEXTOR the main precursor of a disruption is an $m / n=2 / 1$ mode [9]. The duration of the activity of the precursor depends on the plasma parameters. It ranges from several hundred milliseconds to less than one millisecond (two precursor oscillations) before the energy quench phase. The frequency of the mode is $f \approx 2 \mathrm{kHz}$ at the beginning and its amplitude $\tilde{T}_{\mathrm{e}} \approx 30 \mathrm{eV}$. The island width is estimated to $w \leqslant 0.01 \mathrm{~m}$ when the mode becomes visible on the ECE diagnostic. During the evolution towards the disruption the amplitude increases to $\tilde{T}_{\mathrm{e}} \approx 220 \mathrm{eV}$ and the frequency decreases to $f \approx 50 \mathrm{~Hz}$ or the mode even locks. Therefore, we investigated whether one can use this mode as an indicator for a disruption and develop a method to detect the mode.
The method used is based on the dynamic crosscorrelation measurement [5] of two channels of the standard ECE diagnostic located on the HFS and the LFS of the plasma at the $q=2$ surface. For a safe indication of the precursor, the sampling frequency of the analog to digital converter in the module is $50 \mathrm{kHz}$. The cross-correlation coefficient is calculated from the high pass filtered ECE signals. The length of the time window for the cross-correlation calculation can be varied from $0.04 \leqslant t_{\mathrm{w}} \leqslant 20.48 \mathrm{~ms}$. Two radially separated channels are necessary to discriminate between an even mode activity and oscillations of the horizontal plasma position, which yield similar oscillations on the ECE channels. However, with the use of an LFS and a HFS channel such oscillations in the plasma position will be $180^{\circ}$ out of phase, when the cross-correlation technique is applied. At TEXTOR we use the channels with $f=105$ and $145 \mathrm{GHz}$ as input for the correlation module. The ECE diagnostic is, besides the standard magnetic diagnostic and the $\mathrm{HCN}$ interferometer, the most sensitive diagnostic for the detection of large scale MHD modes and it is operated routinely for every TEXTOR discharge. Since the heterodyne ECE diagnostic is a very localized measurement of the mode it is sensitive enough to detect the mode rather early. With a video bandwidth of $10 \mathrm{kHz}$ the measurement allows to detect modes according to the Nyquist theorem up to $5 \mathrm{kHz}$. A problem is imposed by the good radial resolution of the ECE diagnostic and the high localization of the mode in its early stage. The application, therefore, requires the correct adjustment of the toroidal magnetic field, otherwise the observed EC resonant layers are shifted far from the mode of interest. The crosscorrelation method, however, can also be used with other nonlocalized signals, e.g. magnetic coils.

The cross-correlation module generates a signal in transistor transistor logic (TTL), which, in the case of TEXTOR, triggers the neutral beam injection within $8 \mathrm{~ms}$ after the trigger, or opens a fast helium valve [6] that is used to mitigate the disruption effects on the plasma facing components. The set-up of the whole system is shown in figure 1 in combination with the neutral beam injector. The neutral beam injectors can be operated in two different modes. (i) constant $1 \mathrm{~s}$ injection and (ii) injection for the duration of the trigger only. The time for the detection of the mode varies between 1.5 and $25 \mathrm{~ms}$ depending on the amplitude of the mode and its position with respect to the observation volume of the ECE diagnostic. The trigger onset can be controlled by the

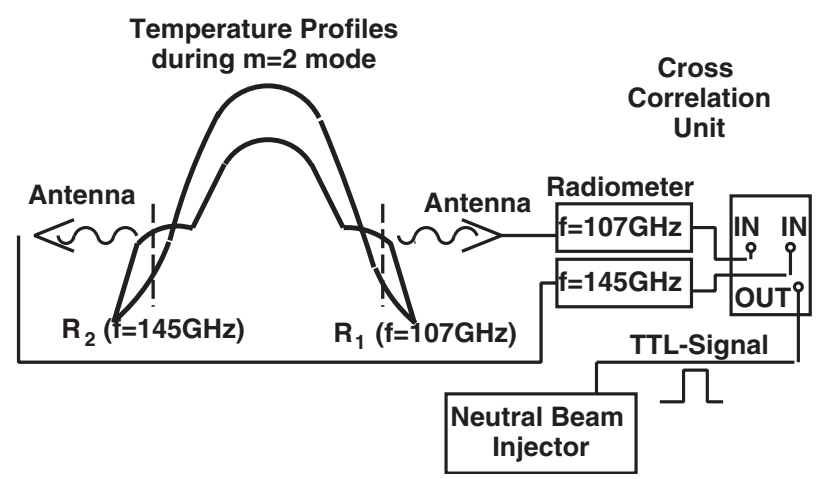

Figure 1. Set-up of the cross-correlation mode detector and connection to the neutral beam injection control at TEXTOR. 
threshold for the cross-correlation coefficient. The use of a large coefficient moves the trigger onset closer to the disruption and makes it more difficult to avoid a disruption, whereas a small coefficient not only increases the probability of a false alarm but also increases the probability to avoid a disruption.

\section{Reliability of the mode detection}

The system uses two heterodyne ECE radiometers with a high spatial localization, $\Delta r=0.01 \mathrm{~m}$, of the standard electron temperature diagnostic at TEXTOR. The detection of an $m=2$ mode can only be achieved for standard values of magnetic field, plasma current and horizontal plasma position. Deviations in the mode position due to horizontal shifts of the plasma column or different plasma currents can be tolerated, but the time needed to detect the mode is increased due to the missing or bad overlap between the ECE observation layer and the position of the precursor in its early stage. The detection module was operated at TEXTOR from August 2000 until the shutdown in March 2001. During this time more than 1000 discharges were performed with a toroidal magnetic field in the range $2.2 \leqslant B_{\mathrm{T}} \leqslant 2.3 \mathrm{~T}$. It was found from the duration of the discharges that $\approx 15 \%$ of the performed discharges at TEXTOR ended with a disruption. Not all of the disruptions were recognized by the cross-correlation technique. Some plasmas showed only small or very short $m / n=2 / 1$ mode activity. In these cases, our method was not fast enough to detect the precursor. A trigger was generated in 264 discharges, 152 of which ended in a disruption. For 112 plasmas a trigger was generated but the discharge continued (figure $2(a)$ ). This shows that the trigger was also generated in discharges that does not disrupt at all. These are mainly plasmas with a stationary $m / n=2 / 1$ mode.

In $58 \%$ of the disruptions the trigger was generated by mode activity, long lasting modes and also by mode bursts. For some discharges the trigger was too late to avoid a disruption. However, the trigger could still be used to mitigate disruption

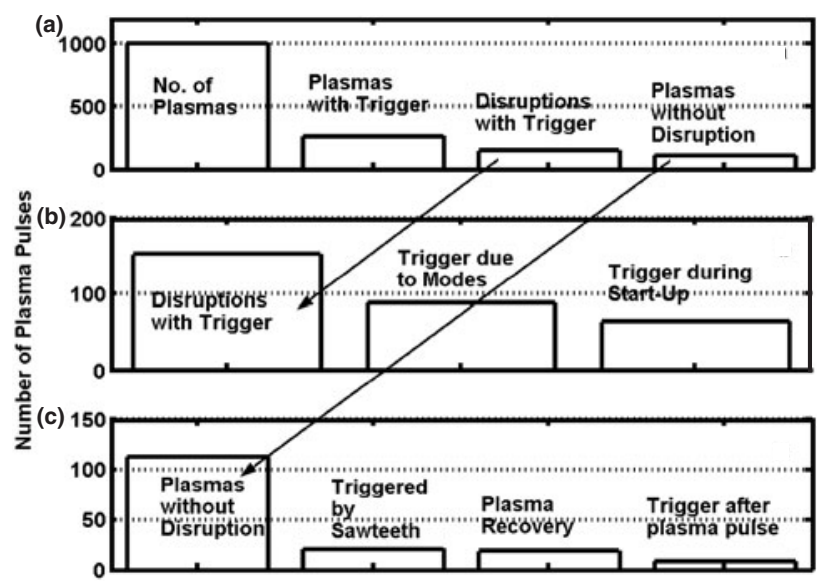

Figure 2. Statistical analysis of 6 months operation: (a) the number of performed discharges for $2.2 \leqslant B_{\mathrm{t}} \leqslant 2.3 \mathrm{~T}$ and the fraction with the generation of a trigger. Column 3 is the number of disruptions and trigger and column 4 those plasmas with trigger and no disruption. (b) Shows the amount of plasmas with mode activity and those that disrupt within $t \leqslant 0.1 \mathrm{~s}$ generating a trigger. (c) Some reasons for trigger generation in non-disrupting plasmas. effects by He gas puffing. Due to plasma start-up conditions the trigger was generated for 64 plasmas within $t \leqslant 100 \mathrm{~ms}$ (figure $2(b)$ ). These plasmas disrupted in the early start phase due to wrong gas pre-fill programmes or failures in the position and current control. In these cases the trigger was generated by the fast and correlated rise of the radiation temperature observed by the ECE channels, used as input in the crosscorrelation module.

From the 112 discharges having a trigger without disruption (false alarm), some were low density discharges with strong additional neutral beam heating and large inverted sawteeth at the $q=2$ surface. They showed a fast, in phase temperature increase followed by a slow decay on LFS and HFS, respectively. This caused the module to generate a trigger as well. They contributed $19 \%$ to the total number of discharges with a trigger (figure $2(c)$ ). For $17 \%$ the plasmas were recovered from the mode. These plasmas belong mostly to the programmes for disruption avoidance where additional neutral beam heating was applied to avoid the disruption. In $8 \%$ the trigger was generated at the plasma end by a fast temperature decrease.

Neglecting those discharges where the plasma disrupts within $100 \mathrm{~ms}$ and where disruptions occurred without a preceding mode, the module was quite successful. For the dedicated programmes the success rate of the disruption avoidance and mitigation was up to the order of $50 \%$. However, one has to keep in mind that these programmes were performed in spite of finding the operational limits of the method. Therefore, the success rate can only be a first rough estimate and not representative for a regular tokamak operation. The reliability investigations also showed that a detection of the $m / n=2 / 1$ mode is not enough to decide if a plasma will drive towards a disruption. It needs further measurement, e.g. the island growth rate, to reduce the number of plasmas with false alarm.

\section{Development of modes during the disruption precursor phase}

\subsection{Mode generation in the plasma start-up phase}

For the analysis of the MHD modes prior to a disruption the ECE diagnostic is mainly used. Furthermore, with a charge exchange recombination spectroscopy (CXRS) diagnostic the toroidal plasma rotation is analysed from the velocity distribution of particle species. At TEXTOR this diagnostic can only be applied together with tangential co-injection of neutrals. The line emission of impurities in the VUV range is observed with a Spred spectrometer.

To prove the $m=2$ mode detector on its functionality, plasma scenarios were developed to generate the disruption precursors deliberately. Therefore, the electron density was increased by gas puffing between $0.2 \leqslant t \leqslant 0.5 \mathrm{~s}$ and simultaneously the current rise after the start-up phase of $t \approx 100 \mathrm{~ms}$ was retarded. After $600 \mathrm{~ms}$ the flat top plasma current was achieved (figures $3(a)$ and $(b)$ ). Due to the small plasma current and the additional gas puffing, the plasma edge cooled down. This favoured the excitation of MHD instabilities. With this method, the mode activity could be seen already for $t \approx 300 \mathrm{~ms}$ after plasma start on the central 
channels of the ECE diagnostic (see figure 3(c)). In all the discharges analysed, with the above described start-up conditions, the mode activity started when the plasma current crossed the value $I_{\mathrm{p}}=240 \mathrm{kA}$ at $\bar{n}_{\mathrm{e}}=1.3 \times 10^{19} \mathrm{~m}^{-3}$. This is more than a factor two smaller than the expected density limit in stationary discharges with the same plasma current and line averaged density [10]. The analysis of the ECE data at this time showed the first appearance of an even mode deep in the plasma core at the ECE channels of $R=1.97$ and $1.62 \mathrm{~m}$, corresponding to a flux surface radius of $\rho=0.18 \mathrm{~m}$. Calculations with a standard current profile of the type $j=$ $j_{0}\left(1-(\rho / a)^{2}\right)^{3}$ yields $q=2.1$ at this radius. ECE channels located well outside the $q=2$ surface saw no mode activity. The development of the $q=2$ surface is shown in figure 4 for the discharge 90314 assuming a Shafranov-shift on the axis of $s_{0}=0.03 \mathrm{~m}$. The plasma current and the horizontal plasma position, deduced from interferometric measurements, needed for the calculations were taken from the experiment.

(a)

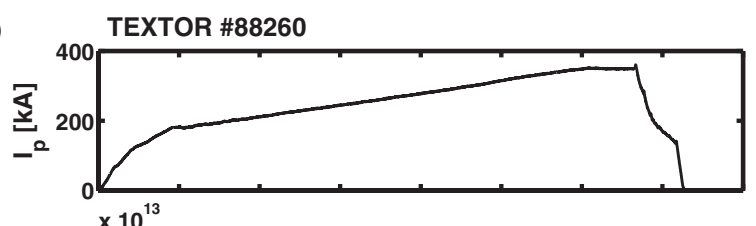

(b)

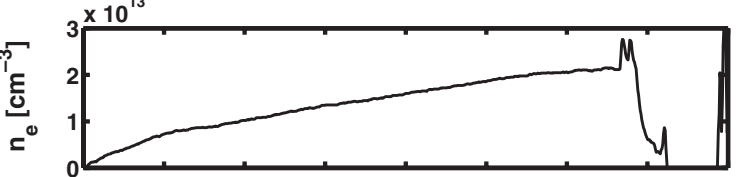

(c)

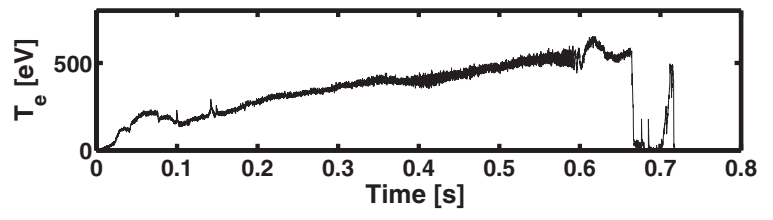

Figure 3. Reproducible mode generation at TEXTOR. The three upper signals show the development of $I_{\mathrm{p}}$ and $\bar{n}_{\mathrm{e}}$ for the mode generation.

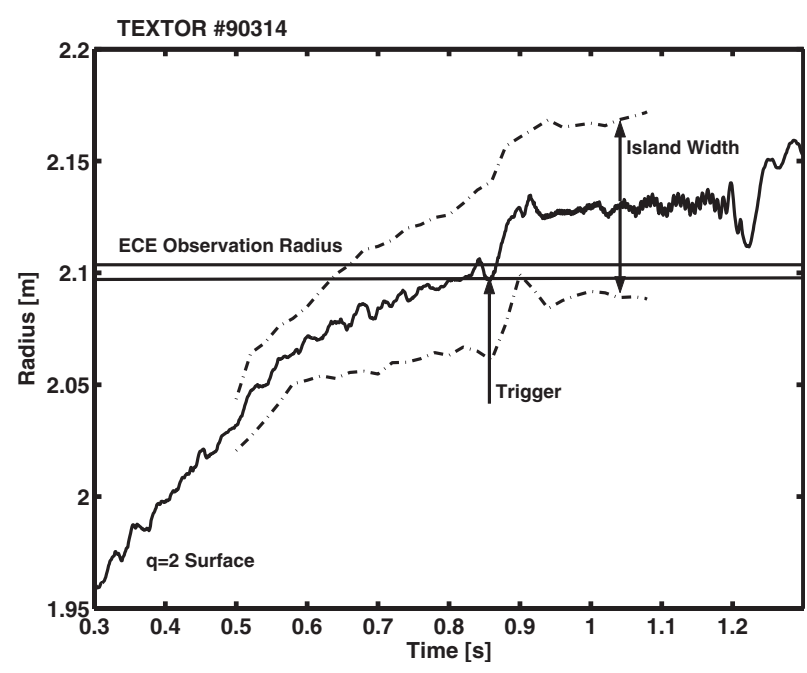

Figure 4. Development of the $q=2$ surface. The horizontal lines mark the radial observation region of the ECE radiometer.

Furthermore, the estimated island width is included and shows the increase of the island width in the plasma.
The island width can be approximated by the equation for a symmetric island

$$
\frac{w}{2} \simeq \frac{\tilde{T}_{\mathrm{max}}}{\nabla T_{\mathrm{e}}}
$$

where $\tilde{T}_{\max }$ is the largest measured amplitude of the mode oscillation and $\nabla T_{\mathrm{e}}$ the mean electron temperature gradient in the time interval of interest. At $t \approx 0.6 \mathrm{~s}$ a large radial region of the plasma is already modulated by the island and intersects on the LFS with the observation volume of the ECE channel used for the cross-correlation. The corresponding ECE channel on the HFS was not yet effected by the mode and hence the cross-correlation was still zero and the trigger not generated. At $t \approx 0.8 \mathrm{~s}$ the $q=2$ surface touched the outermost ECE channels at $R=2.10$ and $1.52 \mathrm{~m}$ for the first time. The cross-correlation coefficient exceeded its preprogrammed value and a trigger was generated. The onset of the trigger was also visible in the offline cross-correlation calculations, performed for the mean subtracted ECE channels at $R=2.10$ and $1.52 \mathrm{~m}$, shown in figure 5. The trigger started at $0.865 \mathrm{~s}$ (position of the arrow in figure 5), just at the time where the cross-correlation coefficient exceeded the pre-programmed threshold for the first time. The further development of the cross-correlation was nicely seen. The appearance of a second maximum at a time lag of $2.5 \mathrm{~ms}$ corresponding to a mode frequency of $400 \mathrm{~Hz}$ was interesting. It appeared around $t=0.9 \mathrm{~s}$ and was due to the correlation of the signals shifted by one period of the mode. From the crosscorrelation calculations the decrease of the mode frequency was seen from the increasing time lag for the second maximum. Also, a minor disruption at $t=1.25 \mathrm{~s}$ was seen. At this time the correlation between the ECE channels vanished completely.

The precursor phase of pulse 90311 is shown in figure 6 . In figure $6(a)$ the evolution of the mode frequency, deduced from the ECE channel at $R=2.02 \mathrm{~m}$, towards a disruption is shown for the ECE channel at $R=2.02 \mathrm{~m}$. At $t \approx 0.6 \mathrm{~s}$ the $q=2$ surface intersected with the observation radius of the

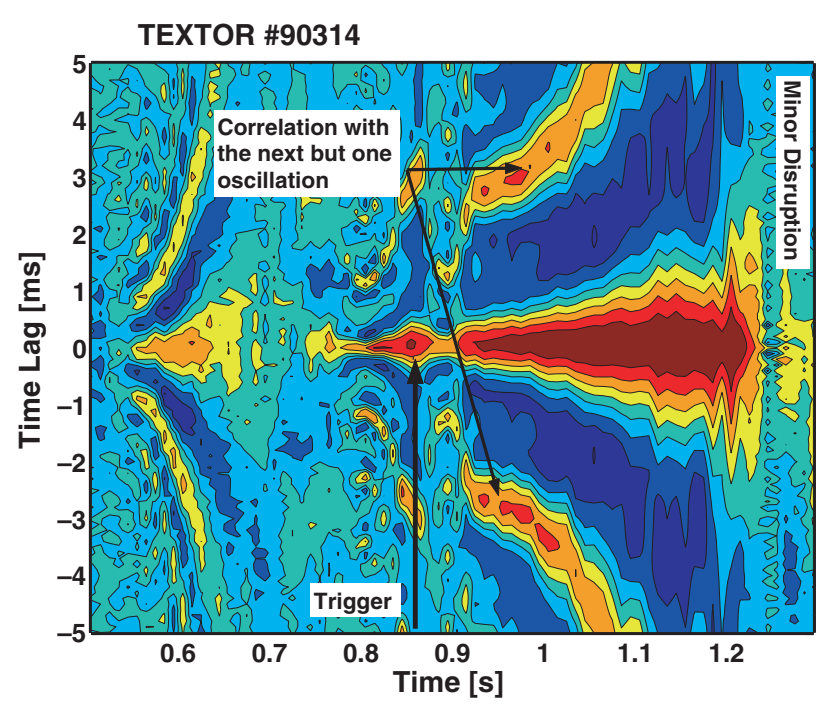

Figure 5. The cross-correlation for the radiometer at $R=1.52$ and $2.10 \mathrm{~m}$. A first significant correlation is obtained for $t \geqslant 0.865 \mathrm{~s}$. The second maximum in the cross-correlation shows the decrease of the mode frequency. At $t=1.25 \mathrm{~s}$ the correlation disappears due to a minor disruption. 
ECE channel at $R=2.06 \mathrm{~m}$ and $R=1.58 \mathrm{~m}$, respectively. These channels showed a clipping and a doubling of the mode frequency as expected [11].

The neighbouring ECE channels on the LFS at $R=2.02$ and $2.10 \mathrm{~m}$ were $180^{\circ}$ out of phase (figure $6(b)$ ). An island width $w=0.05 \mathrm{~m}$ was estimated (see figure 4 and equation (1)). At the outermost ECE channel on the LFS, used for the mode detection, the mode appeared at $t \approx 0.6 \mathrm{~s}$ with a frequency $f \approx 500 \mathrm{~Hz}$. At this time, the $q=2$ island had not yet reached the observation radius of this ECE channel; however, the temperature oscillations due to the magnetic perturbation, resulting from the island formation, could be measured even outside the separatrix of the mode. In addition, it was found from the amplitude of the oscillations that the island was asymmetric since the inner side of the separatrix showed an amplitude of $\Delta T_{\mathrm{e}}=120 \mathrm{eV}$ whereas the amplitude on the outer side yields only $\Delta T_{\mathrm{e}}=40 \mathrm{eV}$.

At $t=0.78 \mathrm{~s}$ the clipping appeared on the outermost channels $R=2.10 \mathrm{~m}$ and $1.52 \mathrm{~m}$ (figure 6(c)). The channel at $R=2.06 \mathrm{~m}$ showed no further clipping. A check with the $q=2$ surface from the calculations was again in agreement with the position of the outermost ECE channel. After the short and sudden increase in the mode frequency, which is discussed in section 4.3, the general evolution towards the disruption was characterized by a fast decrease of the mode frequency correlated with an increase of the mode amplitude and mode width. The frequency decrease shown in figure $6(a)$ resulted in a mode locking and a minor disruption where a part of the plasma energy was released to the wall. The process of mode locking followed by a minor disruption could be repeated several times until the plasma disrupted finally. In the given case two minor disruptions were observed for $t \approx 1.1 \mathrm{~s}$ (figure $6(a)$ ). During the mode evolution no signs of a beginning major disruption were observed in the plasma current and line averaged density. Negative spikes, indications for a minor disruption, were observed on the loop voltage

\section{TEXTOR \#90311}

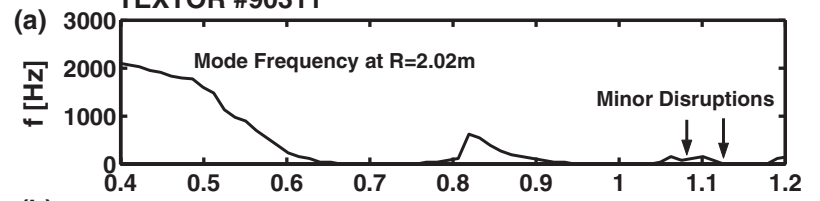

(b)
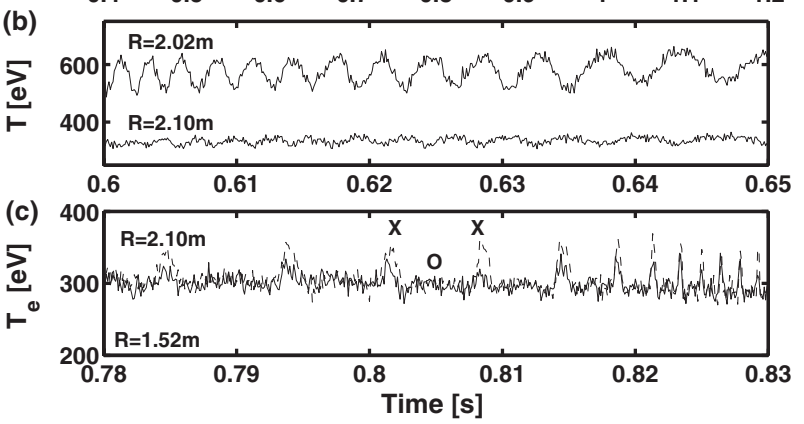

Figure 6. Evolution of modes towards a disruption as seen by different ECE channels for pulse \#90311. (a) Evolution of the $m / n=2 / 1$ frequency, arrows indicate minor disruptions. (b) The $m / n=2 / 1$ mode observed by ECE at $R=2.02$ and $2.10 \mathrm{~m}$, showing that the mode is located between both channels. $(c)$ The $m / n=2 / 1$ island at the outermost ECE channels. Frequency clipping is visible for the whole time interval. shortly before the disruption. This demonstrates that the detection of local changes is necessary for an early disruption detection.

The whole process of the $m / n=2 / 1$ development was accompanied by another mode-like structure in the plasma centre. This structure had the same frequency as the $m / n=$ $2 / 1$ mode but with a different phase relation. A phase difference of $90 \leqslant \Phi \leqslant 120^{\circ}$ was found between the ECE channels located at $R=1.92$ and $1.76 \mathrm{~m}$.

\subsection{Disruption suppression on $\mathrm{OH}$-target plasmas with NBI injection}

Due to the recipe described above, first experiments were performed on Ohmic target plasmas, where a neutral beam injection was triggered by the detection of the disruption precursor.

For a better understanding of the described mechanism the influence of the tangential neutral beam injection on the rotation frequency is reviewed. Co-injection was used as a synonym for the injection in the direction of the plasma current, counter-injection was upstream the plasma current direction. Since the natural mode rotation was in a counterdirection, co-injection decreased the mode frequency and counter-injection increased the mode frequency. The mode frequency was proportional to the plasma rotation, because the mode was frozen in the plasma. The effect of the tangential neutral beam injection was twofold: (i) increasing the limits for instabilities and (ii) creating a large rotation shear that is expected to tear apart the $m / n=2 / 1$ mode.

In figure 7 two discharges are shown demonstrating the success of the method. Both discharges had the same plasma parameters and the same temporal evolution for $I_{\mathrm{p}}$ and the line averaged density $\bar{n}_{\mathrm{e}}$ figures $7(a)$ and $(b)$. Both plasmas showed an $m / n=2 / 1$ mode at $t \approx 0.5 \mathrm{~s}$ (figure $7(c)$ ) and a drop in the central electron temperature (figure $7(d)$ ). The vertical line at $t=0.633 \mathrm{~s}$ marked the onset of the neutral beam injection for discharge \#88264. In this case, the discharge was continued after neutral beam injection and the plasma current was ramped down after $4 \mathrm{~s}$ regularly. The other discharge was stopped by a disruption at $t=0.7 \mathrm{~s}$.

A detailed discussion of the disruption avoidance for discharge \#88265 (see figure 8) is given below. From the figure the development from an MHD unstable plasma to a plasma with sawtooth activity is seen. The $m / n=2 / 1$ mode was first detected by the ECE channel at about $t=0.3 \mathrm{~s}$ at $R=2.02 \mathrm{~m}$. The continuation of the plasma ramp-up phase yield a further outward movement of the mode. At $t=0.5 \mathrm{~s}$ the mode was located between $2.06 \leqslant R \leqslant 2.10 \mathrm{~m}$ on the LFS with a frequency of $f \approx 2 \mathrm{kHz}$ (figures $8(b)$ and $(c)$ ). Already, $50 \mathrm{~ms}$ later the stationary mode started to grow and within $50 \mathrm{~ms}$ its frequency decreased to $200 \mathrm{~Hz}$. During the slowdown of the frequency the island width increased (figure $8(a)$ ). The island width calculated at $t=0.61 \mathrm{~s}$ amounted to $w=0.10 \mathrm{~m}$. The trigger signal was generated at $t=0.603 \mathrm{~s}$ when the $q=2$ surface intersected the ECE channel at $R=1.58 \mathrm{~m}$ on the HFS and $R=2.06 \mathrm{~m}$ on the LFS. Already, $8 \mathrm{~ms}$ after the generation of the disruption trigger neutral beam, with a power $0.5 \leqslant P_{\mathrm{NBI}} \leqslant 0.7 \mathrm{MW}$, is co-injected. In the plasma core, indications for an $m / n=1 / 1$ mode were found at the same 
frequency as the $m=2$ mode close to the minor disruption. For the whole profile the temperature increased and the mode was locked. This is the consequence of the co-injection, which decreased the velocity and its shear by flattening the rotation profile. A minor disruption was observed at $t=0.644 \mathrm{~s}$. After the minor disruption the mode activity started at $t=0.655 \mathrm{~s}$ but disappeared quite fast. Also, the island width decreased rapidly. The mode structure seems to be complex and a superposition of an $m / n=2 / 1$ and an $m / n=3 / 2$ mode. The first sawtooth appeared at $t=0.687 \mathrm{~s}$, indicating the existence of a $q=1$ surface in the plasma. The sawtooth activity is seen in figure $8(d)$ where the sawtooth crashes are represented by the periodic high frequency bursts, when performing a sliding fast

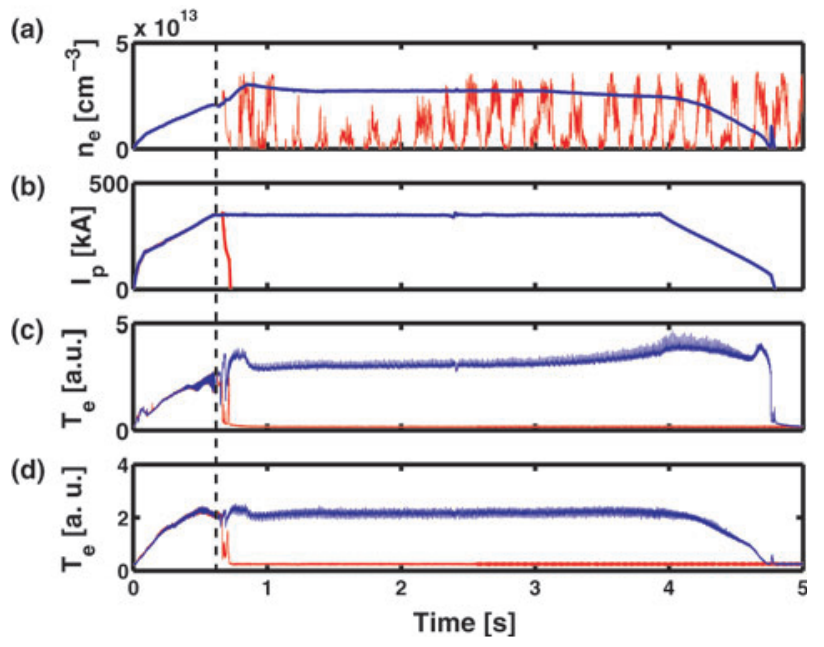

Figure 7. Comparison of two discharges with (blue \#88264) and without (red \#88260) neutral beam injection. (a) Line averaged density. (b) Plasma current. (c) ECE channel at $R=2.1 \mathrm{~m}$. $(d)$ Central ECE channel. The dashed vertical line marks the onset of the tangential neutral beam injection.

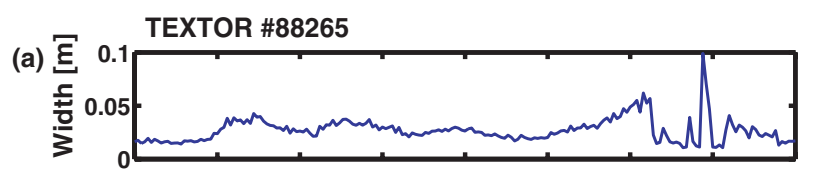

(b)
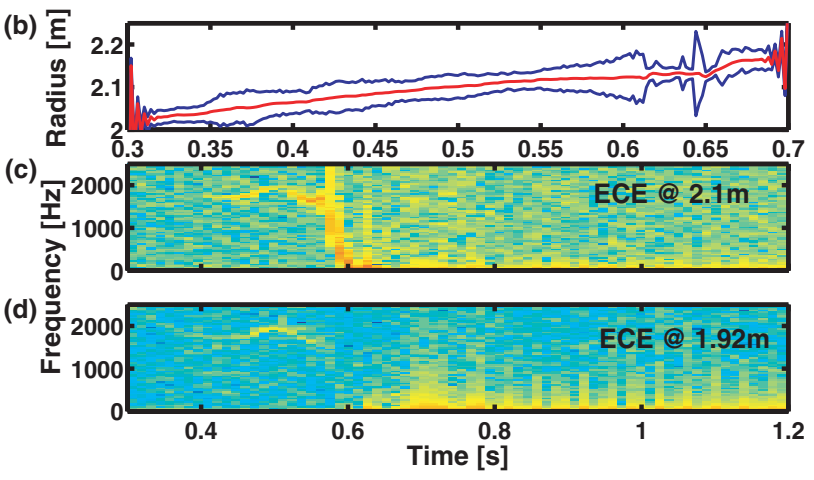

(d)

Figure 8. Disruption avoidance in an $\mathrm{OH}$ target plasma. (a) The island width of the $m / n=2 / 1$ mode. (b) The position of the $q=2$ surface and the island width showing the growing island. (c) Spectogram of the outermost ECE channel on the LFS showing the mode (yellow) evolution. $(d)$ The appearance of the sawtooth activity is monitored by the spectogram of a central ECE channel at $R=1.92 \mathrm{~m}$. Note that the time axis has changed for subplots $(a),(b)$ and $(c),(d)$
Fourier transformation on the time interval of interest. With the extinction of the $m / n=2 / 1$ mode, the cross-correlation coefficient dropped below the pre-programmed threshold. As a consequence, the injector was switched-off. Nevertheless, the sawtooth activity persisted and the plasma continued until the normal current ramp down.

This behaviour was quite reproducible for $0.5 \leqslant P_{\mathrm{NBI}} \leqslant$ $0.7 \mathrm{MW}$. For smaller or larger values of $P_{\mathrm{NBI}}$ plasma disrupts. The lower limit can be explained by the missing toroidal momentum. On TEXTOR about $P_{\mathrm{NBI}} \approx 0.2 \mathrm{MW}$ was necessary to stop the mode rotation. An additional power of $0.3 \mathrm{MW}$ was necessary to build up the required velocity shear. In one case with $P_{\mathrm{NBI}}=0.51 \mathrm{MW}$, the $m / n=2 / 1$ mode could be stabilized with co-injection and rotated at $f=3.7 \mathrm{kHz}$, whereas in the plasma centre sawteeth are observed. The upper limit for the added co-injection power may be explained by the additonal perturbation level induced by NBI injection, in an already disrupting plasma.

\subsection{Disruption suppression on auxiliary heated plasmas with NBI generated velocity shear}

In order to avoid the flattening of the toroidal rotation profile when co-injection was applied alone, both co- and counterinjectors were used in the same plasma with different power and therefore with different angular momentum input. It has been observed that co-neutral beam injection $\left(P_{\mathrm{NBI}} \approx 200 \mathrm{~kW}\right)$ flattens the toroidal plasma rotation profile and increases the width of the mode. This observation was applied to enhance the growing rate of the mode. An additional advantage of the early co-injection was the possibility to monitor the plasma rotation by CXRS in the precursor phase of the disruption.

In figure 9, radial profiles of the toroidal plasma rotation are shown for two discharges with and without counterinjection. Concerning the main plasma parameters and mode evolution, both shots are similar up to the time where counterinjection is started by the trigger. To obtain large disruption precursors co-injection was applied at $T=0.5 \mathrm{~s}$ in both discharges. In the co-injection phase a flattening of the

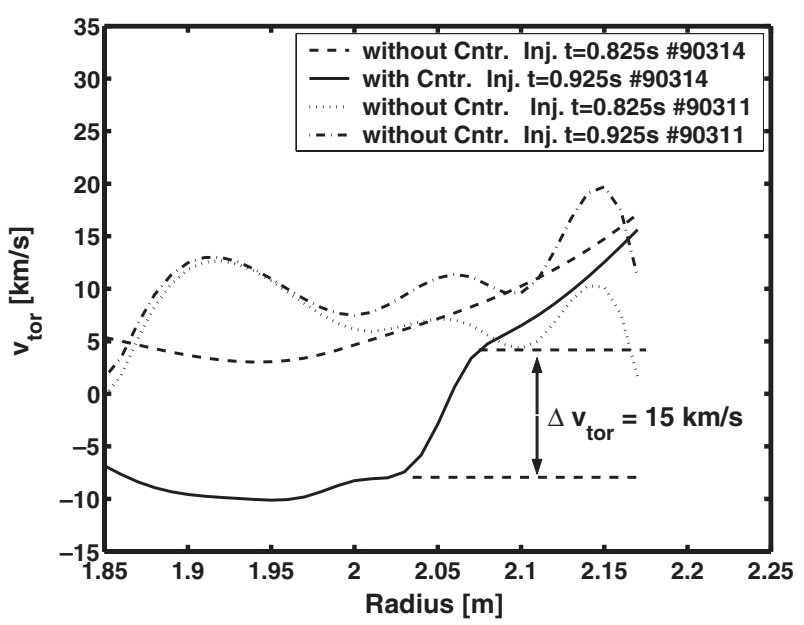

Figure 9. Influence of the counter-injection on toroidal rotation profiles. The step in the rotation profile with onset of the counter-injector takes place in the vicinity of the $q=2$ surface and prevents the mode locking. 


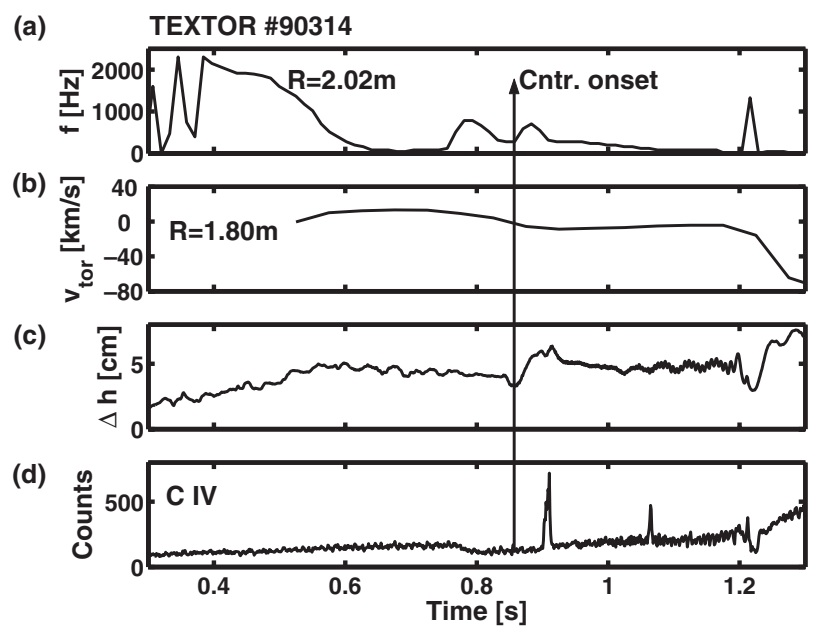

Figure 10. (a) Evolution of the mode frequency as observed by an ECE channel at $R=2.02 \mathrm{~m}$. (b) The toroidal velocity deduced from CXRS at $R=1.80 \mathrm{~m}$. It decreases only slightly with the onset of the counter-injector. After a minor disruption, $\approx 1.2 \mathrm{~s}$, it decreases to $-80 \mathrm{~km} \mathrm{~s}^{-1}$. (c) The horizontal plasma position deduced from interferometry measurements shows an outward movement of the plasma by $\approx 0.03 \mathrm{~m}$ with the onset of the counter-injection. (d) C IV emission line exhibits increase due to plasma wall contact at $t=0.9 \mathrm{~s}$.

rotation profile was observed, showing an overall velocity of $7-13 \mathrm{~km} \mathrm{~s}^{-1}$. In the case where the counter-injector is switched-on $\left(P_{\mathrm{NBI}}=0.8 \mathrm{MW}\right)$, a step of $\Delta v_{\text {tor }} \approx 15 \mathrm{~km} \mathrm{~s}^{-1}$ in the rotation profile appeared at $R=2.08 \mathrm{~m}$. This is close to the position of the $q=2$ surface. This step remains for the whole slowing down time of the mode towards the minor disruption and seems to reduce the fast decrease of the mode frequency as observed in plasmas without additional counter-injection, which leads to mode locking followed by a disruption.

A typical discharge with plasma recovery due to counter injection is shown in figure 10. The trigger was generated $50 \mathrm{~ms}$ after the $q=2$ surface intersects the ECE channels used in the cross-correlation calculation. After the activation of the counter-injector at $t=0.865 \mathrm{~s}$ (marked by an arrow in figure 10), the mode frequency increased (figure 10(a)) and the horizontal plasma position moves outward by $0.03 \mathrm{~m}$ (figure $10(c)$ ), shifting the $q=2$ surface to $R=2.13 \mathrm{~m}$. The central plasma rotation (figure $10(b)$ ) decreases slowly and remains nearly constant at $v_{\text {tor }}=-10 \mathrm{~km} \mathrm{~s}^{-1}$ up to the minor disruption, whereas the rotation at $R=2.10 \mathrm{~m}$ remains at $v_{\text {tor }}=10 \mathrm{~km} \mathrm{~s}^{-1}$. It should be mentioned that due to the poor time resolution of the CXRS $(50 \mathrm{~ms})$ fast changes cannot be followed on a timescale, relevant for the MHD changes in the plasma. In the example the plasma moves slowly outward and touches the belt limiter at $t=0.905 \mathrm{~s}$. This causes an inward impurity flux as monitored by the $\mathrm{C}$ IV line (figure $10(d)$ ). Also, other impurity lines such as CaXVIII, CrXxII and Tixx showed an enhancement at that time. This suggests that the $Z_{\text {eff }}$ value is increased. Simultaneously, a drop in the $T_{\mathrm{e}}$ is observed on the outermost ECE channels of $\Delta T_{\mathrm{e}} \approx 100 \mathrm{eV}$. At the same time the $n_{\mathrm{e}}$ profile becomes very broad. The shape turned to a parabolic-like profile with large gradients at the LFS and HFS. The increase of the mode frequency stops with the impurity influx. It is assumed that the additional impurity influx prevents a further increase of

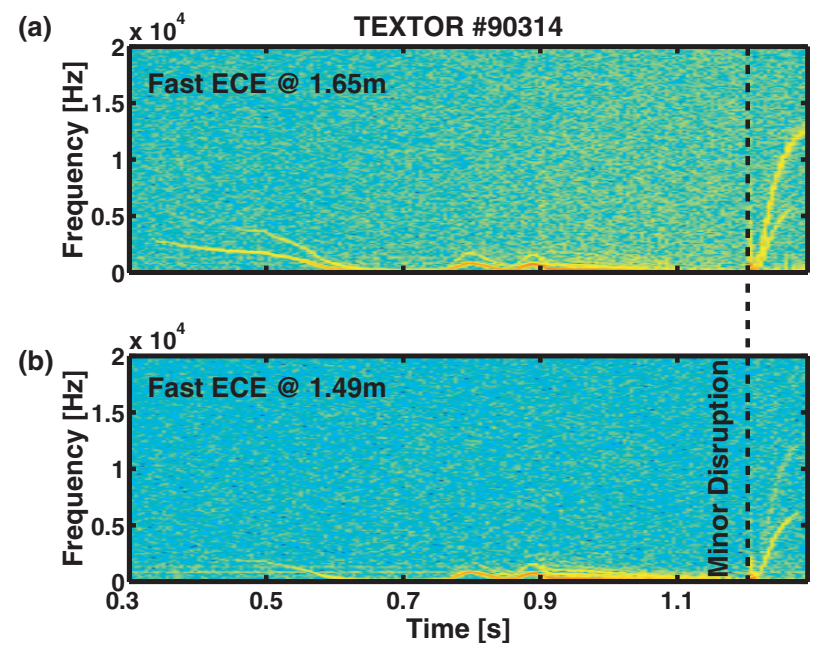

Figure 11. The mode evolution on two fast ECE channels.

(a) Central channel displaying the increase of the mode frequency (up to $15 \mathrm{kHz}$ ) after the minor disruption (- - - ) and

(b) ECE channel outside the $q=2$ surface showing the $7 \mathrm{kHz}$ mode.

the mode frequency and leads to a frequency slowdown within $300 \mathrm{~ms}$. In this phase harmonics of the mode frequency are observed on several ECE channels indicating the large size of the $m=2$ island. In the plasma centre temperature oscillations with the same frequency as the $m / n=2 / 1$ mode appear. From the phase relation of the oscillation a $m / n=1 / 1$ mode was excluded. At TEXTOR a missing $m / n=1 / 1$ mode in a sawtooth free plasma is always an indication for an increased impurity contents and $Z_{\text {eff }}$, respectively [12]. After a minor disruption at $t=1.2 \mathrm{~s}$, modes appear again. On several fast ECE channels (resolution $\Delta t=1 \mu \mathrm{s}$ ) we observe two modes. In the centre, the mode frequency increases up to $15 \mathrm{kHz}$, whereas the frequency increases up to $7 \mathrm{kHz}$ at the outermost ECE channel (figure 11). This central mode has been observed with $\mathrm{O}$ mode reflectometry on the LFS too. The appearance of the mode is correlated with a decrease to the central toroidal rotation to $v_{\text {tor }}=-80 \mathrm{~km} \mathrm{~s}^{-1}$. Already, $50 \mathrm{~ms}$ after the minor disruption the first sawtooth appears in the plasma centre. Within the next $50 \mathrm{~ms}$ the plasma exhibited decreasing mode as well as an increasing sawtooth amplitude. With the disappearance of the modes the plasma's profile changed and smooth gradients developed across the profile. The described development, sequencing the minor disruption was similar for all analysed discharges. After $t=1.9 \mathrm{~s}$ the injector was switched-off and a rotation profile returning back to normal was observed.

A peculiarity when using co-injection to flatten the rotation profile was an increase of the mode frequency within $t \leqslant 40 \mathrm{~ms}$, as seen in figure $10(a)$, by the small hump at $t=0.78 \mathrm{~s}$. This feature was observed in many discharges. It lasted for $t \leqslant 100 \mathrm{~ms}$. To estimate the temporal evolution of the mode frequency we calculated the different contributions according to the equation

$$
f_{\text {mode }}=\frac{m \cdot v_{\Theta}}{2 \pi \cdot r} \pm \frac{n \cdot v_{\Phi}}{2 \pi \cdot R_{0}}+f_{\text {dia }}
$$

where $f_{\text {dia }}$ is the diamagnetic frequency that depends on the electron pressure gradient $\nabla p_{\mathrm{e}}, v_{\Theta, \Phi}$ are the poloidal and 
toroidal velocities and $m, n$ are the toroidal and poloidal mode numbers, which are in the case of interest $m=2$ and $n=1$. For the calculations, the toroidal velocity and the ion temperature were taken from the CXRS measurements. The poloidal magnetic field was calculated from a standard current density profile. The contribution of the beam driven current could be neglected since the heating power $P_{\mathrm{NBI}}=$ $150 \mathrm{~kW}$ was small. The pressure gradient was calculated from the $T_{\mathrm{e}}$ and $n_{\mathrm{e}}$ profiles measured by the ECE diagnostic and the HCN interferometer, respectively. The radius used in the calculations was set to the measurement position of the ECE channel at $R=2.06 \mathrm{~m}$ and transformed to flux surface geometry. Furthermore, for the calculation of the poloidal velocity, the neoclasical approach [13] was used:

$$
v_{\Theta}=k \cdot \frac{c}{e B_{\Phi}} \nabla T_{\mathrm{i}}
$$

with all the quantities in cgs units. The coefficient $k$ depends on the regime where the measurements are performed [14]. In the special case, the calculations were performed between the banana and plateau regimes; therefore, the coefficient was interpolated between both the regimes. Furthermore, $v_{\Theta}$ depended on $\nabla T_{\mathrm{i}}$, which was estimated from CXRS data. The calculations were performed for discharge \#90314 on a time grid of $\Delta t=0.05 \mathrm{~s}$, which was the time resolution of the CXRS diagnostic. The calculated mode frequency is plotted in figure 12 together with the $m / n=2 / 1$ mode frequency of the ECE channel at $R=2.06 \mathrm{~m}$. For the calculations we assumed $Z_{\text {eff }}=1$ for $t \leqslant 0.9 \mathrm{~s}$. As soon as the plasma touched the limiter and the impurity contents increased, $Z_{\text {eff }}=2$ was assumed. In the range $0.5 \leqslant t \leqslant 0.7 \mathrm{~s}$ the decrease of the mode frequency was well described and experiment and calculations were in good agreement. The increase in the mode frequency at $t \approx 0.8 \mathrm{~s}$ was correlated with a decrease in the frequency of the poloidal velocity component due to change in $\nabla T_{\mathrm{i}}$. For this time a change in the ion temperature profile was observed, which led to the observed increase in the mode frequency. The other two components showed no changes. With the onset

TEXTOR \#90314

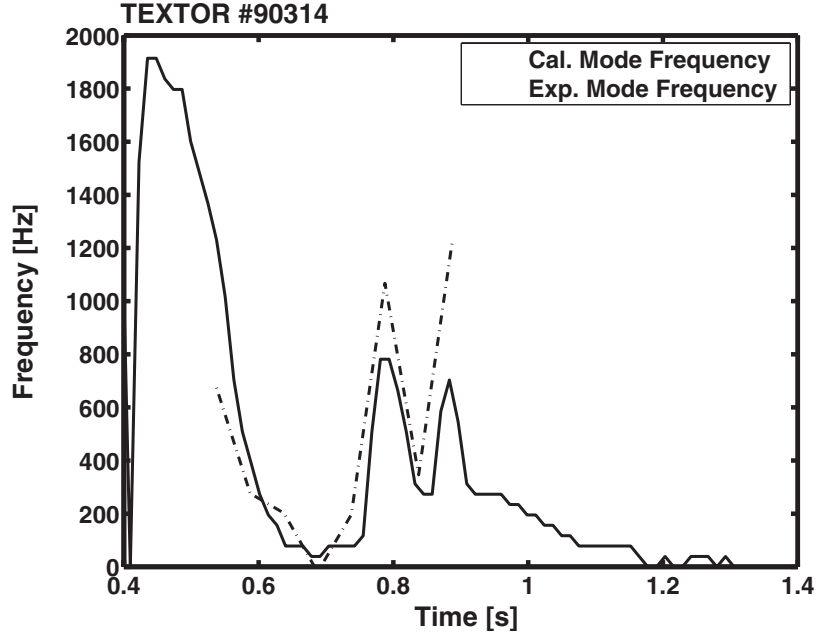

Figure 12. Comparison between measured and calculated mode frequencies. Good correlation between the sudden frequency increase at $t=0.75 \mathrm{~s}$ and the decrease in the toroidal velocity component is seen. of the counter-injector, the diamagnetic frequency changed suddenly, even taking into account an increased $Z_{\text {eff }}$. The frequency of the toroidal velocity component decreased less than expected. The diamagnetic frequency and the frequency of the poloidal velocity component were not changed much. This resulted in a further increase of the mode frequency, which was in agreement with the observed mode frequency with additional counter-injection.

\subsection{Disruption mitigation by He gas puffing}

The main aim of this method was the suppression of runaway electrons that are created during a disruption [15]. Noble gas
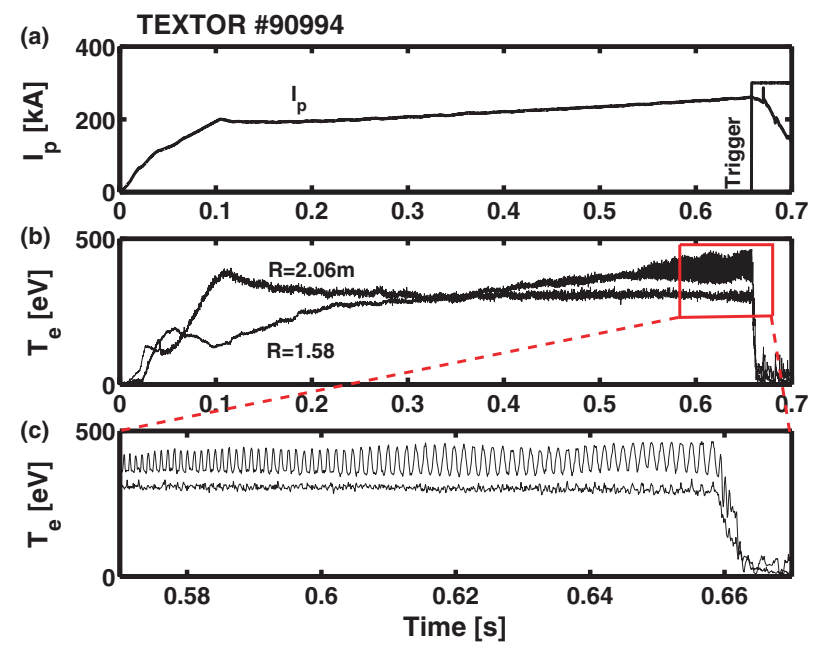

Figure 13. Disruption mitigation with massive He gas puff. (a) Plasma current and trigger signal for the discharge showing the late trigger generation. (b) Time traces of two ECE channels at $R=2.06$ and $1.58 \mathrm{~m}$. (c) Enlarged view showing the mode activity on the LFS channel and the late mode onset on the HFS caused by the late radial extension of the $q=2$ surface. Shortly after the in-phase oscillation of the mode on the HFS, the trigger is fired.

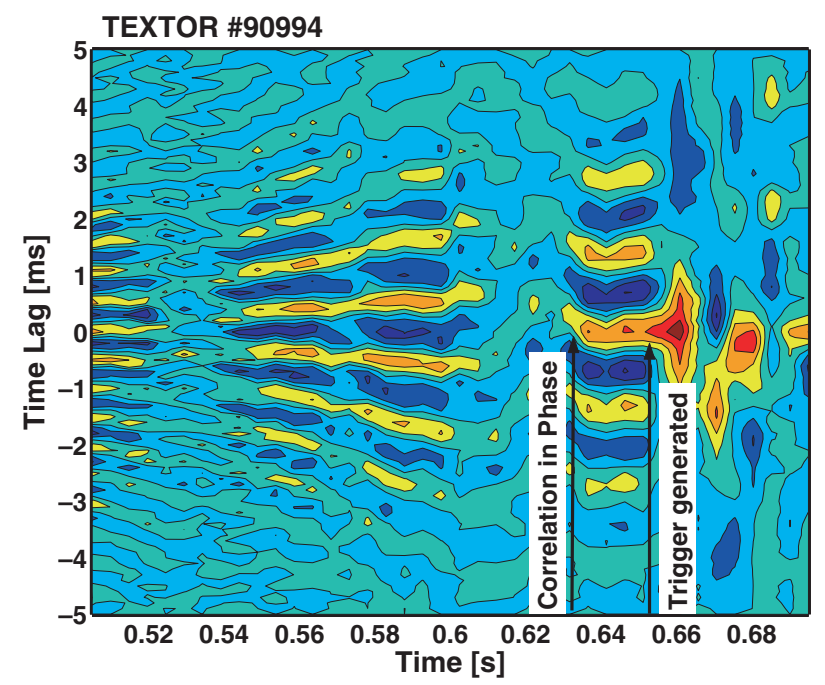

Figure 14. Cross-correlation coefficient for ECE channels at $R=2.06$ and $1.58 \mathrm{~m}$. Up to $t=0.6 \mathrm{~s}$ the coefficient is negative (oscillations are out of phase). After a radial extension of the $q=2$ surface both ECE channels are in phase. Arrows denote the time where the mode oscillation is in phase and the time where the trigger is generated. 
He was used because it can be easily pumped away by the turbomolecular pumps of the belt limiter and because of its low $Z$ number. Furthermore, it had only a small probability to be stored in the wall. The injection of up to $10 \mathrm{mbar}$ litre of helium into the TEXTOR vessel was possible in less than $1 \mathrm{~ms}$ with a valve that uses an eddy current scheme where a pancake coil is adjacent to an aluminium stem. This valve was directly mounted on the TEXTOR vessel. Assuming sound speed propagation, the gas reached the plasma within $1 \mathrm{~ms}$. To simulate the suppression of runaway electrons low density discharges were performed. An example of a discharge terminated by He injection is shown in figure 13 . for pulse \#90994. The mode appeared first on the LFS at $R=2.06 \mathrm{~m}$. The trigger was delayed because the mode appears late $(t=0.645 \mathrm{~s})$ on the HFS. Within $t=15 \mathrm{~ms}$ the trigger was fired and the valve opened immediately. The horizontal periodic structure of hills and valleys in the crosscorrelation coefficient, calculated offline from the two ECE channels (figure 14), showed that modes are evident for $t=0.5 \mathrm{~s}$. However, the cross-correlation exhibited a phase change of $180^{\circ}$ (minimum in the cross-correlation coefficient at zero time lag) between the channels, since both were on different sides of the $q=2$ surface. Up to $t \approx 0.6 \mathrm{~s}$ the mode frequency decreased only slightly. The radial extension of the $q=2$ surface resulted in a reduction of the correlation coefficient for $0.6 \leqslant t \leqslant 0.63 \mathrm{~s}$. Only when at $t \leqslant 0.63 \mathrm{~s}$ did the radius of the $q=2$ surface extend, that is, both ECE channels were well correlated. Furthermore, the example shows that even a large difference in the amplitude of the oscillation on LFS $\left(\Delta T_{\mathrm{e}}=80 \mathrm{eV}\right)$ and $\operatorname{HFS}\left(\Delta T_{\mathrm{e}}=25 \mathrm{eV}\right)$ is tolerable. The injection of He decreased the thermal quench time and therefore the generation of super thermal electrons [7].

It was found that after disruptions with $\mathrm{He}$ gas puffing the following discharge always showed the same good start-up behaviour as the previous one. Due to the no resonant charge exchange of He with the majority gas the bombardment of the wall by high energetic particles was strongly reduced. This allowed a better density control in the start-up phase of the next discharge.

\section{Summary and outlook}

The paper demonstrates the possibility to detect disruption precursors and to avoid or mitigate disruptions using two cross-correlated ECE channels on the HFS and LFS of the plasma. The system demonstrated its reliability within 6 months of TEXTOR operation. The cross-correlation module can be applied to every fusion device, as long as the disruption precursors are identified and the necessary diagnostic is installed. Our method, up to now, was successful in $50 \%$ of the discharges dedicated to disruption studies. The investigations also showed that the method to detect a disruption leads to false alarm in the case of stationary modes.

The module is quite flexible and there are plans to use it also with other mode sensitive diagnostics, e.g. magnetic probes. Furthermore, other mechanisms to avoid or mitigate a disruption are foreseen. With the restart of TEXTOR the use of ECRH for a local heating of the island is also planned.

The injection of co- as well as counter-neutral beam to avoid the disruptions with the onset of a trigger was successfully tested and a detailed analysis of the mode development presented. The measured rotation profiles show the development of a step in the toroidal velocity in the vicinity of the $q=2$ surface, which delays or even prevents a plasma disruption. However, other methods modifying the rotation profile locally will also help to prevent a disruption, e.g. by local tangential momentum input at the plasma edge only. Furthermore, detailed analysis of the frequency development of the $m / n=2 / 1$ mode could explain the observed sudden increase in the mode frequency in discharges with low power co-injection prior to the disruption by changes in the poloidal velocity component.

\section{References}

[1] Federici et al 2001 Nucl. Fusion 411967

[2] Wroblewski D. et al 1997 Nucl. Fusion 37725

[3] Pautasso G. et al 2001 Nucl. Mater. 290-293 1045

[4] Zehetbauer T. et al 2001 Fusion Eng. Des. 56-57 721

[5] Krämer-Flecken A. et al 2001 Fusion Eng. Des. 56-57 773-6

[6] Mank G., Krämer-Flecken A. and Finken K.H. 2001 Mitigation and avoidance of disruptions at TEXTOR-94 28th EPS Conf. on Controlled Fusion and Plasma Physics (Funchal Madeira, 18-22 June 2001)

[7] Finken K.H. et al 2001 Nucl. Fusion 411651

[8] Waidmann G. et al 1997 Rev. Sci. Instrum. 68492

[9] Waidmann G. and Quangli G. 1992 Nucl. Fusion 32645

[10] Waidmann G. 1998 Trans. Fusion Technol. 33 91-8

[11] Fitzpatrick R. 1995 Phys. Plasmas 2 825-38

[12] Rapp J. et al 1997 Plasma Phys. Control. Fusion 39 1615-34

[13] Bugarya V I et al 1985 Nucl. Fusion 25 1707-17

[14] Hazeltine R.D. 1974 Phys. Fluids 17

[15] Gill R.D. 1993 Nucl. Fusion 331613 